\title{
Evolving Agents in a Market Simulation Platform - a Test for Distinct Meta-Heuristics
}

\author{
Naing Win Oo and Vladimiro Miranda, Senior Member, IEEE
}

\begin{abstract}
This paper presents a comparison in performance of 3 variants of Genetic Algorithms (GA) vs. 2 variants of Evolutionary Particle Swarm Optimization (EPSO), made in the extremely complex context of a multi-energy market simulation where the behavior of energy retailers is observed. The simulations are on JADE, a FIPA compliant platform based on intelligent autonomous agents running in a cluster of PCs. Each agent formulates its strategy by an inner complex simulation process using a meta-heuristic that tries to define optimum decisions. The results suggest that an EPSO approach is more efficient than GA.
\end{abstract}

Index Terms-Energy Markets, Autonomous Agents, Genetic Algorithms, Particle Swarm Optimization

\section{INTRODUCTION}

$\mathrm{T}$ he comparison of the performance of distinct metaheuristics surfaces from time to time and is useful in providing guidance both to researchers and developers of practical applications. Meta-heuristics are particularly promising in complex problems where no tractable mathematical model may be used.

A truly complex problem is the realistic simulation of energy markets, allowing insight on how different market structures and regulation policies behave in reality. This understanding cannot be achieved only by theoretical models, lacking the ability to analyze the micro-interaction among actors in complex markets due to their complexity [1]. This simulation may be achieved through building a platform based on intelligent autonomous agents.

One of the fundamental steps that gave intelligent agent technology its seniority has been the work developed under FIPA, the Foundation for Intelligent Physical Agents [2], whose specifications served as the basis for much progress in several areas. FIPA specifications have become standards and open source software platforms are now available that are FIPA compliant. Among these, we have selected for our work the platform JADE - Java Agent Development Framework [3][4]. This option allows portability, independent development of agents, standardization of the development process and modularity; it benefits from all the knowledge in FIPA specifications and the successes of their application in other areas such as telecommunication.

Not many applications are known of Intelligent Agents (IA) in the domain of Power Systems. An important and early one has been developed inside project ARCHON, under the ESPRIT Research Programme of the European Union [5]. The idea of using agents to simulate markets has already been proposed [6] and recent work also reports the use of IA in electricity market simulation [7].

Naing Win Oo is with INESC Porto, Portugal (nwo@inescporto. pt). Vladimiro Miranda is with INESC Porto, Portugal and also with FEUP, Faculty of Engineering of the University of Porto, Portugal (vmiranda@inescporto.pt).
Considering multiple energy markets and not only electricity is a must nowadays, because in many countries the businesses of electricity and of gas have become intertwined with cross interests, as a consequence of the development of conversion technologies such as microturbines or fuel cells. In the near future, if not now, a consumer may choose to have his power supply assured by the gas supplier via some terminal energy conversion equipment. Moreover, micro CHP installations allow users to sell power to the grid while assuring heating at home and this option is already available currently for large buildings. In colder countries we may consider also competition with district heating. This competition effect will be especially felt at distribution level, and what seemed impossible in the electricity business is a reality when one considers a multi-energy market: parallel distribution networks of electricity, gas and heat that develop side by side in the attempt to gain consumers. The concept of natural monopoly, applicable to a single energy sector, loses meaning in this context.

The work reported in this paper demanded the independent specification of Agents representing entities in the market. Each agent has its own objectives, internal processes of decision and form of communication with other agents. Among them, retailers receive a special focus, because we have given them the ability to evolve and adapt to market conditions. This capacity is conferred by an internal procedure based on a meta-heuristic procedure. A Retailer Agent will, from time to time, proceed to perform an internal simulation of the evolution of the market in order to try to forecast market changes and optimize its own moves (like defining selling prices) to beat competition (by improving profits, gaining market share, etc).

This is an extremely complex optimization problem and, therefore, constitutes an attractive challenge for testing distinct algorithms. Some preliminary results were presented in [8] and this paper discusses new results, comparing Genetic Algorithms with EPSO -Evolutionary Particle Swarm algorithms, in distinct simulation context definitions. These results again confirm what has independently and recently been confirmed by Japanese researchers [9], that EPSO algorithms exhibit a superior performance.

\section{The Market And ITS Agents}

For a multi-energy market simulation, we have developed a model with nineteen Agents. This model is simple and does not intend to represent any particular real market, but to serve as a conceptual test bed. Some of the functions actually implemented just mimic real world circumstances, but the modular concept behind the Agent technology allows one to replace any function by a more sophisticated one without disturbing model architecture. The 19 Agents and their basic functions are: 
A - Economy - This agent translates into energy demand variables basic data such as economic drive, season of the year, weather conditions. These demand values are passed to the Information Environment Agent.

B - Consumer - Agents (Residential, Commercial and Industrial) of this type do not represent individual consumers, but groups of consumers such as residential, commercial or industrial. Each agent purchases a mix of energies and changes market shares of these energies according to prices, needs, elasticity of demand and adjustment delays to price changes. Energy efficiency and costs of capacity increments are also taken in account.

C - Information Environment - This agent acts as a blackboard where market actors post information regarding their current actions and request information for evaluating new actions. Besides communication, it also performs compilation on the data obtained from market participants, providing more clear and transparent information.

D - Energy Retailer - Retailer Agents exist for electricity (2), gas (2) and heating. Every agent of this type has as internal functions a) monitoring its performance to achieve profitability as well as market share control, b) finding optimal decisions for performance improvement, and c) improving management efficiency.

One important function inside a Retailer agent is strategic planning through a "simulation inside the simulation" process, based on the best knowledge an agent has of the behavior of the others. In our model these agents use neural networks to predict consumption and prices; and evolutionary computing to plan ahead an optimal strategy for expansion of the business and price determination.

E - Delivery - These agents (electricity, gas and heating) perform duties such as extending networks over the territory to supply new consumers. Network expansion is performed using functions optimizing paths and profits, which are also available in GIS platforms. An agent of this type has a logic of its own and also seeks to maximize profit while guaranteeing contracts of supply. Competing for new territory is one of the functions implemented and therefore investments are also weighted against running profits.

F - Regulator - The regulatory agents (electricity, gas and heat) impose simple restrictions such as limiting the duration between successive price changes and imposing price-caps over prices of energy. More complex actions are under observation.

G - Market Operator - These agents (electricity, gas and heat) acts as the replacement of wholesale market, issuing day-ahead energy prices.

During a simulation cycle, each market Agent performs the tasks mentioned above rotationally and the cycle ends when every market actor finishes performing its duties.

\section{IMPLEMENTATION}

Our multi-energy market simulation platform has been developed over JADE (Java Agent DEvelopment Framework), a FIPA compliant distributed multi-agent software framework based on the peer-to-peer communication architecture, fully implemented in Java. Its adoption simplified the implementation of multi-agent systems. The intelligence, the initiative, the information, the resources and the control can be fully distributed on mobile terminals as well as on computers in a fixed network [3]. The scheduling of agents is made with a hybrid of sequential and parallel processes. The sequential scheduling is adopted at entity or class level, where agents wait for their turn to receive updated information. For an entity having multiple agents, a parallel agent scheduling approach is used, to take advantage of parallel processing.

The agents are represented in Fig. 1. The platform has been run on a cluster of 5 PCs connected in parallel. Agents are arranged such that no two parallel-scheduling agents under an entity exist on the same computer. Note that the Information Environment Agent is at the central PC.

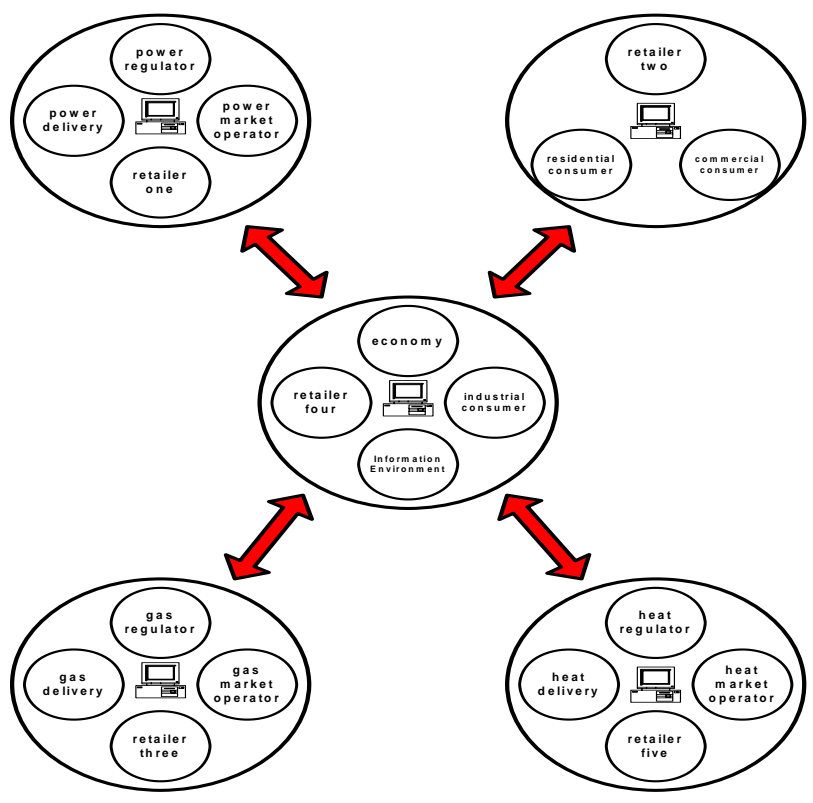

Fig. 1. Parallel arrangement of 19 Agents in a cluster of 5 PCs

\section{The RetAiLer AgenT}

\section{A. General concept}

Each Energy Retailer entity periodically monitors movements in market share and profitability, evaluates its own economical performance, and defines its operational state, either profit-taking or share-taking, depending on whether the fall in profit or market share is beyond an allowable limit, or normal state otherwise. Then, the decision process of manipulating influential variables such as energy prices, incentives, publicity, service, quality and management efficiency is the object of an internal optimization procedure based on evolutionary computation.

The general fitness function ruling the internal process is

$$
\text { Max } O B J=\sum_{d=1}^{n}\left[\text { Eco }_{d}-\text { Pen }_{d}\right]
$$

(1)

where: $\mathrm{Eco}_{\mathrm{d}}=$ Economical performance at day d

$$
\text { Pen }_{\mathrm{d}}=\text { Penalty assigned at day } \mathrm{d}
$$

Each time this decision process is called for, an internal simulation of the evolution of the market along a period of $n$ days is performed. The Agent seeks to optimize its decisions for the following period and bases its simulation on the best estimation it has of demand and the behavior of other agents. Also, the Energy Retailer who requires more energy than the amount bought with contracts will buy from the wholesale market, represented by the Market Operator 
Agent for each type of energy. For these estimations, we have implemented a neural network procedure to predict consumptions and prices based on recent market evolution. This mechanism has been included in every retailer so they have been put in similar circumstances. This way, we expect to explain the differences in performance of Retailer Agents, when equipped with distinct meta-heuristics, as a function of the effectiveness of the algorithms themselves.

Simulations were based on time steps that we've called "days"; an internal simulation in a Retailer Agent extended for two "months" or "60 days", after which the fitness function is evaluated for any candidate set of decisions. The decision that leads to the best result in the end of the optimization process is accepted as the decision to be taken for current situation. The decision-making duration, which is the delay time in the decision-making process, was set at 14 "days" and candidate variables come to effect after that.

\section{B. Some details of the model}

In this section we will present the general ideas behind the retailer agent model. Because of space constraints, many details will be missing but may be found in [10]. In general terms, the optimization model implemented inside a Retailer Agent has the form of (1), subject to a number of constraints in costs, reliability and incentives given to clients, whose meaning is believed to be clear:

$$
\begin{aligned}
& \text { Price }_{\mathrm{a}}^{\min }<\text { Price }_{\mathrm{a}}<\text { Price }_{\mathrm{a}}^{\max } \\
& \text { Deviation _ in _ Pr ice } \text { a }_{2}<\text { Limit } \\
& \text { Incentive }{ }_{\mathrm{a}}^{\min }<\text { Incentive } \mathrm{a}<\text { Incentive }{ }_{\mathrm{a}}^{\max } \\
& \text { Advertise }{ }_{\mathrm{a}}^{\min }<\text { Advertise }{ }_{\mathrm{a}}<\text { Advertise }{ }_{\mathrm{a}}^{\max } \\
& \text { Service } \underset{\mathrm{a}}{\min }<\text { Service }{ }_{\mathrm{a}}<\text { Service } \underset{\mathrm{a}}{\max } \\
& \text { Quality }{ }_{\mathrm{a}}^{\min }<\text { Quality }_{\mathrm{a}}<\text { Quality }{ }_{\mathrm{a}}^{\max } \\
& \text { Management }{ }_{\mathrm{a}}^{\min }<\text { Management } \mathrm{a}<\text { Management } \max _{\mathrm{a}}
\end{aligned}
$$

The objective function is a combination of two parts, economical performance and penalty assigned for breaking rules. The economic performance was evaluated using the following formula.

$$
\text { Eco }_{d}=\mathrm{P}_{\mathrm{d}} \times \mathrm{UP}_{\mathrm{d}}+\mathrm{S}_{\mathrm{d}} \times \mathrm{US}_{\mathrm{d}}
$$

where

$$
\begin{aligned}
& U_{d}=\text { Unit profit of retail at day d } \\
& U_{d}=\text { Unit market share at day d } \\
& P_{d}, S_{d}=\text { Profit, share weight factor at day d }
\end{aligned}
$$

The economic performance of the retailer is judged by two components, its profit and market share holding. The profit is evaluated after the moves of all agents in the market at each time step and depends not only on the decisions of a Retailer but on all the decisions taken by all the other agents. The market share is a consequence of the reaction of Consumer Agents to prices and other marketing effects (such as incentives).

The energy retailer is designed to take different actions in different situations. When the action foreseen is of the aggressive type in which attention is only fixed on profiting, setting $\delta \mathrm{S}, \delta \mathrm{P}$ as deviations in market share or prices, the economical performance is evaluated by

$$
\mathrm{Eco}_{\mathrm{d}}=\left\{\begin{array}{c}
\mathrm{P}_{\mathrm{d}}^{1} \times \mathrm{UP}_{\mathrm{d}}+\mathrm{S}_{\mathrm{d}}^{0} \times \mathrm{US}_{\mathrm{d}} \quad \text { if } \delta \mathrm{P}_{\mathrm{d}} \geq 0 \\
\mathrm{P}_{\mathrm{d}}^{2} \times \mathrm{UP}_{\mathrm{d}}+\mathrm{S}_{\mathrm{d}}^{0} \times \mathrm{US}_{\mathrm{d}} \quad \text { if } 0>\delta \mathrm{P}_{\mathrm{d}} \geq \delta \mathrm{P}_{\text {limit }}^{\text {lower }} \\
\mathrm{P}_{\mathrm{d}}^{3} \times \mathrm{UP}_{\mathrm{d}}+\mathrm{S}_{\mathrm{d}}^{0} \times \mathrm{US}_{\mathrm{d}} \quad \text { if } \delta \mathrm{P}_{\mathrm{d}}<\delta \mathrm{P}_{\text {limit }}^{\text {lower }}
\end{array}\right\}
$$

subject to $\quad \mathrm{P}_{\mathrm{d}}^{0}<\mathrm{P}_{\mathrm{d}}^{1}<\mathrm{P}_{\mathrm{d}}^{2}<\mathrm{P}_{\mathrm{d}}^{3}$.
When the action is defensive, in which attention is more on market share gaining, the economical performance is evaluated as

$$
\text { Eco }_{d}=\left\{\begin{array}{c}
P_{d}^{0} \times U_{d}+S_{d}^{1} \times U_{d} \quad \text { if } \delta S_{d} \geq 0 \\
P_{d}^{0} \times U_{d}+S_{d}^{2} \times U_{d} \quad \text { if } 0>\delta S_{d} \geq \delta S_{\text {limit }}^{\text {lower }} \\
P_{d}^{0} \times U_{d}+S_{d}^{3} \times U_{d} \quad \text { if } \delta S_{d}<\delta S_{\text {limit }}^{\text {lower }}
\end{array}\right\}
$$

subject to: $\quad \mathrm{S}_{\mathrm{d}}^{0}<\mathrm{S}_{\mathrm{d}}^{1}<\mathrm{S}_{\mathrm{d}}^{2}<\mathrm{S}_{\mathrm{d}}^{3}$

Penalties are evaluated as follows when an aggressive move is foreseen:

$$
\text { Pen }_{\mathrm{d}}=\mathrm{C}+\delta \mathrm{S}^{2} \times \mathrm{F}
$$

and as follows when the action prepared is defensive

$$
\text { Pen }_{\mathrm{d}}=\mathrm{C}+\delta \mathrm{P}^{2} \times \mathrm{F}
$$

where $\mathrm{C}$ are constants and $\mathrm{F}$ are penalty factors.

The following mathematical model is used for adjusting consumer consumption to the movement of commodity prices, giving new market shares:

$$
{ }_{C} S_{p}^{d}={ }_{C} S^{d} \times \frac{\left(\mathrm{CP}_{p}^{d}\right)^{-k}}{\sum_{i=1}^{n}\left(C P_{i}^{d}\right)^{-k}}
$$

where

$$
\begin{aligned}
& { }_{\mathrm{C}} \mathrm{S}_{\mathrm{p}}^{\mathrm{d}}=\text { Share of commodity p in consumer type c at day d } \\
& { }_{\mathrm{C}} \mathrm{S}^{\mathrm{d}}=\text { Share of consumer type } \mathrm{c} \text { at day d } \\
& \mathrm{CP}_{\mathrm{p}}^{\mathrm{d}}=\text { Converted price of commodity p at day d } \\
& \mathrm{CP}_{\mathrm{i}}^{\mathrm{d}}=\text { Converted price of commodity i at day d } \\
& \mathrm{k} \quad=\text { Attitude factor of consumer type c on commodities }
\end{aligned}
$$

To incorporate time delay on consumption adjustment to commodity price changes, the following formula has been used so that the shares of commodities are gradually changed during the delay span

$$
\mathrm{C}_{\mathrm{p}}^{\mathrm{d}}=\mathrm{LS}_{\mathrm{p}}^{\mathrm{i}} \pm \frac{\mathrm{SC}_{\mathrm{p}}}{\pi}\left[\operatorname{ArcTg} \frac{\mathrm{t} \times \mathrm{C}}{\mathrm{t}_{\mathrm{m}}^{2}-\mathrm{t}^{2}}\right]
$$

where

$\mathrm{LS}_{\mathrm{p}}^{\mathrm{i}}=$ Limit to share change for commodity $\mathrm{p}$ in condition i

$\mathrm{SC}_{\mathrm{p}}=$ Share change of commodity $\mathrm{p}$ due to price change

$\mathrm{t}_{\mathrm{m}}=$ Mid-point of delay time

$\mathrm{t}=$ Time between commodity price change and present

$\mathrm{C}$ = Coefficient factor of share changing

Coefficient $k$ en (7) determines the degree of rigidity of the market in reaction to price changes, coefficient $C$ in (8) determines how fast the market adapts to price changes. Demand elasticity, a parameter that influences consumer decisions regarding the level of energy consumption, is related with these coefficients and derived from factors such as quality of commodity, service provided and publicity, and is interpreted as the attitude of a particular consumer towards a particular energy commodity. The demand response to a changing economic environment is set to be lowest in low elasticity and highest in high elasticity.

These market share changes result from decisions inside Consumer Agents and become known by Retailers through interaction with the Environment Agent.

In our experiments, we have fixed $n=60$ days for all internal simulations. In all cases, the stopping criterion has been the same: in the first month, when performing the first 
internal simulation, the evolutionary process would be stopped if after 50 consecutive generations there were no improvement in the fitness function; in all the following internal simulations, during the market simulation of 24 months, we have used the threshold of 10 generations instead of 50 .

\section{EXPERIMENTS}

In a previous paper [8] we have reported some results of competition between two meta-heuristic approaches Genetic Algorithms (in 2 variants) and EPSO, Evolutionary Particle Swarm Optimization (1 variant). This paper enlarges the competition landscape in two ways. First, we include now 3 variants of GA and 2 variants of EPSO. Second, we test the algorithms in three types of scenario: with low, medium and high demand elasticity.

The consideration of these scenarios is relevant because e have demonstrated [10] that the market converges to very different solutions depending on the level of awareness of market conditions by consumers and on the elasticity of their response: with low elasticity we found that Retailer Agents developed coordinated strategies similar to oligopoly and that prices would rise without any visible market share change, while with high elasticity a really competitive market would develop with oscillations in market share and moderation in price raise to the advantage of consumers. See Fig. 2 and Fig. 3 as illustration.

The meta-heuristic variants tested, all using the same fitness function (1), were:

1. GAST: Genetic Algorithm, standard Stochastic Tournament (GA with classical operators)

2. GADC: Genetic Algorithm with Deterministic Crowding as the selection operator

3. GAMP: Genetic Algorithm with Multiple Populations

4. EPSO, with a population with the same size as GA

5. MiniEPSO, with a swarm half the size of EPSO

GA are well known meta-heuristics and we will not describe their nature. In GADC, the Deterministic Crowding operator acts as follows:

a) two individuals are selected randomly

b) by crossover, two new individuals are generated

c) a technique of similarity is applied to the set of two parent and two descendent individuals to group them in two pairs, maximizing the similarity inside each pair; for instance, in chromosomes coding the individuals in bits, one may use Hamming distance (no. of different bits) to classify similarity

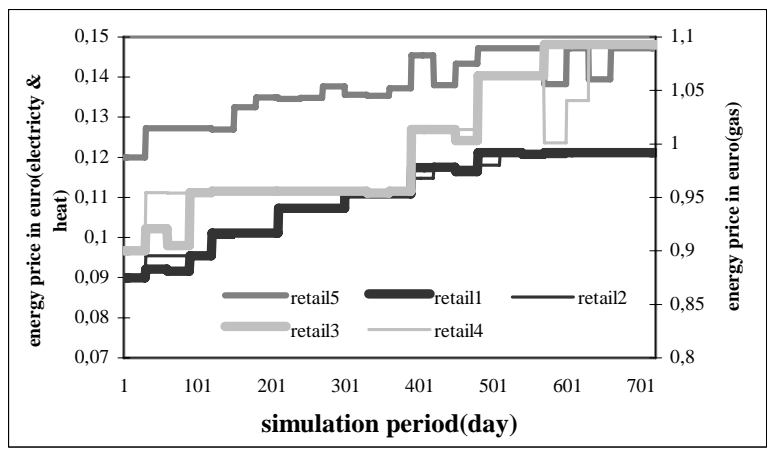

Fig. 2. Evolution of energy retail prices for residential consumers in a test case scenario with low elasticity of demand regarding supply

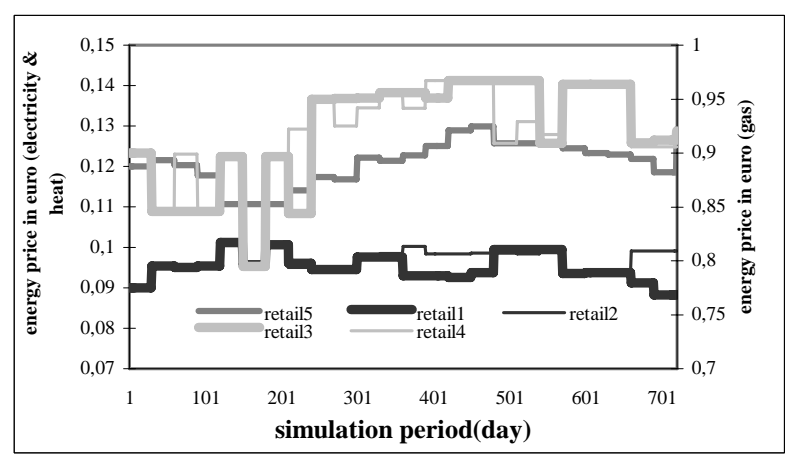

Fig. 3. Evolution of energy retail prices of residential consumer in a test case scenario with high elasticity of demand regarding supply

d) an elitist selection is applied for each group of similar individuals, finally selecting two individuals to form the next generation

e) this process is repeated until the following generation has the desired number of individuals.

In GAMP, we have built a GA with only two subpopulations of equal size; at each step in time we have exchanged two individuals, randomly selected, from one of the sub-populations to the other, before crossover is applied. This strategy (such as DC) is an attempt to avoid the loss of genetic diversity.

EPSO - Evolutionary Particle Swarm Optimization, is a hybrid in concepts of Evolutionary Algorithms and Particle Swarm Optimization, first proposed in [11] and with applications in Power Systems [12]. The reader is referred to these publications because space constraints do not allow its fully developed description. It is an Evolutionary Algorithm (close to the family of Evolution Strategies and Evolutionary Programming) where the mutation operator is only applied to strategic parameters and the recombination operator is non-conventional: it is, in fact, the "movement rule” of PSO (Particle Swarm Optimization) methods.

Recombination is an operation that produces new offspring from some form of combination of parent individuals, chosen in the population (the classical recombination operator, in GA, is called crossover). The movement rule of PSO generates a new individual as a weighted combination of parents, which are: a given individual in the population, the best ancestor of this individual and the best ancestor of the present generation. This may be seen as a form of intermediary recombination. In this type of recombination in evolutionary algorithms, a new individual is formed from a weighted mix of ancestors, and this weighted mix may vary in each space dimension.

The recombination rule for EPSO is the following: given a particle $\mathbf{X}_{i}$, a new particle $\mathbf{X}_{i}^{\text {new }}$ results from

$$
\begin{gathered}
\mathbf{X}_{\mathrm{i}}^{(\mathrm{k}+1)}=\mathbf{X}_{\mathrm{i}}^{(\mathrm{k})}+\mathbf{V}_{\mathrm{i}}^{(\mathrm{k}+1)} \\
\mathbf{V}_{\mathrm{i}}^{(\mathrm{k}+1)}=\mathrm{w}_{\mathrm{i} 0}^{*} \mathbf{V}_{\mathrm{i}}^{(\mathrm{k})}+\mathrm{w}_{\mathrm{i} 1}^{*}\left(\mathbf{b}_{\mathrm{i}}-\mathbf{X}_{\mathrm{i}}\right)+\mathrm{w}_{\mathrm{i} 2}^{*}\left(\mathbf{b}_{\mathrm{g}}^{*}-\mathbf{X}_{\mathrm{i}}\right)
\end{gathered}
$$

where the symbol * indicates that these parameters will undergo evolution under a mutation process, and

$\mathbf{b}_{\mathrm{i}}$ - best point found by the line of ancestors of individual i up to the current generation

$\mathbf{b}_{\mathrm{g}}$ - best overall point found by the swarm of particle in their past life up to the current generation

$\mathbf{b}_{\mathrm{g}}^{*}=\mathbf{b}_{\mathrm{g}}+\mathrm{w}_{\mathrm{i} 4}^{*} \mathrm{~N}(0,1)$ - it is an individual in the neighborhood of $\mathbf{b}_{\mathrm{g}}$. 
$\mathbf{X}_{\mathrm{i}}^{(\mathrm{k})}$ - location of particle $\mathrm{i}$ at generation $\mathrm{k}$

$\mathbf{V}_{\mathrm{i}}^{(\mathrm{k})}=\mathbf{X}_{\mathrm{i}}^{(\mathrm{k})}-\mathbf{X}_{\mathrm{i}}^{(\mathrm{k}-1)}$ - is the "velocity" of particle $\mathrm{i}$ at generation $\mathrm{k}$

$\mathrm{w}_{\mathrm{i} 1}$ - weight of the inertia term (a new particle is created in the same direction as its previous couple of ancestors)

$\mathrm{w}_{\mathrm{i} 2}$ - weight of the memory term (the new particle is attracted to the best position occupied by its ancestors) $\mathrm{w}_{\mathrm{i} 3}$ - weight of the cooperation or information exchange term (the new particle is attracted to the overall best-sofar found by the swarm).

$\mathrm{w}_{\mathrm{i} 4}$ - weight affecting dispersion around the best-so-far

EPSO is a self-adaptive algorithm because the weights that regulate recombination are taken as strategic parameters and are mutated and allowed to evolve. Selection acts on the recombination operator weights and, from generation to generation, a better (adaptive) recombination operation evolves.

Each individual has been assigned with random values at the initial stage. The standard number of individuals in the population was set at 20 (except in miniEPSO) and each individual possessed ten variables. These variables were related with:

- 3 energy prices for residential, commercial and industrial consumers

- 3 financial incentives to residential,, commercial and industrial consumers

- $\quad$ advertisement cost

- $\quad$ service cost

- quality improvement investment

- investment on management efficiency improvement

For all meta-heuristics tested, and to smooth the influence of the random processes, each simulation under each scenario has been run 5 times. The evolution of the market has been simulated for a period of 720 days in all cases and regardless of the method, the same fitness (objective) function and stopping criteria have been used.

\section{RESULTS}

In the following figures we summarize the most interesting results obtained, in the comparison of performance of the five algorithms, used to emulate the internal decision process of a Retailer Agent.

The figures display the profit gained month by month by a Retailer Agent. One of the measures of the success of a strategy is the integral of the curve, which in a way is an indicator of the accumulated profits (not considering interest rates and compound interests).

First of all, Fig. 4 to Fig. 9 confirm what was already suggested by Fig. 2 and Fig. 3: higher elasticity in consumer response leads to reduced profits in Retailers. The reason must be found in the fact that the real market operates more according to an ideal market when the reaction of consumers to price changes is more quick and clear. When for some reason the consumer reaction is too slow, a Retailer may impose higher prices without fear of losing market share.

The other interesting conclusion from this work is that in all scenarios the Retailers equipped with an EPSO algorithm behaved better than those with any of the three variants of Genetic Algorithms.

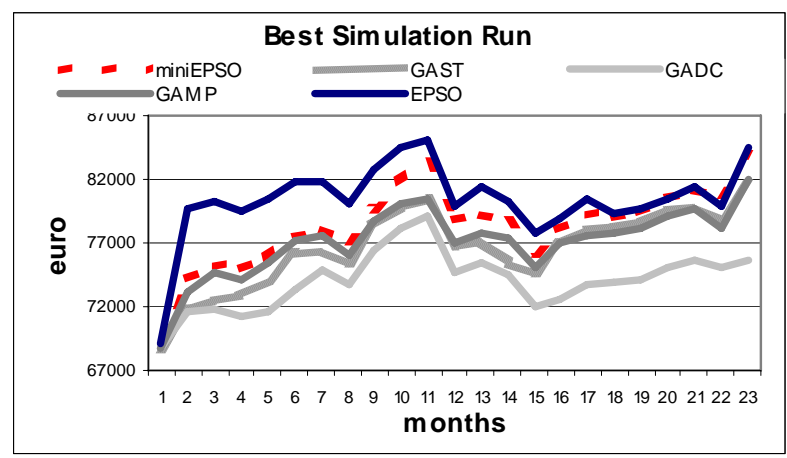

Fig. 4. Evolution of monthly profits for Retailer Agents with different decision engines - best run in 5 attempts for low demand elasticity.

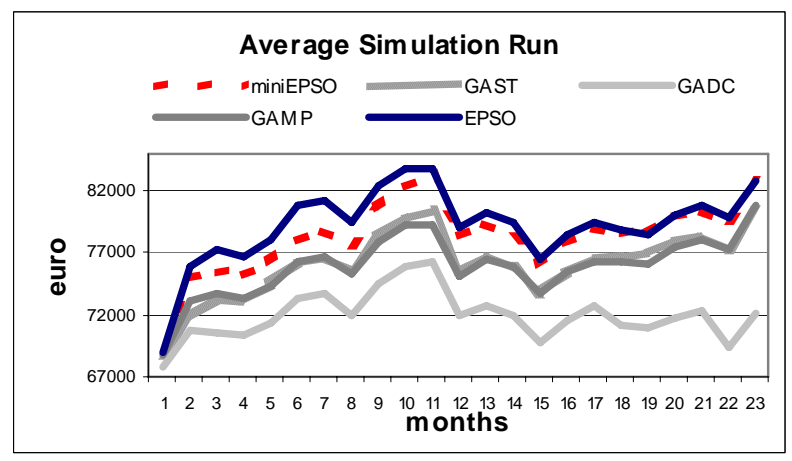

Fig. 5. Average of 5 runs for the evolution of monthly profits for Retailer Agents with different decision engines - low demand elasticity.

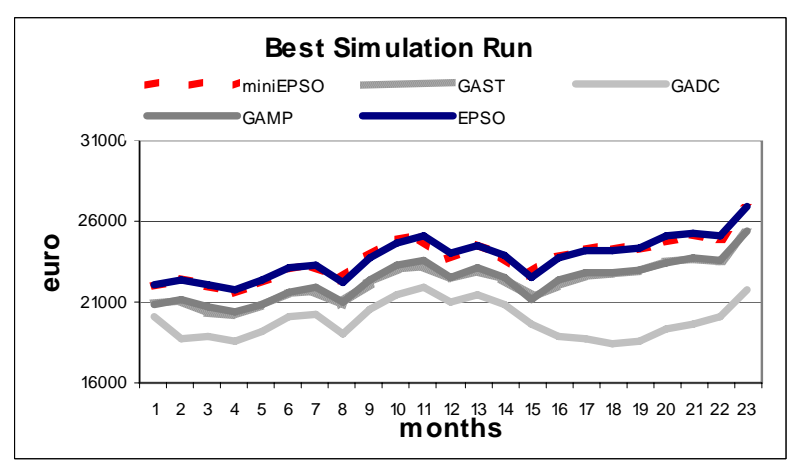

Fig. 6. Evolution of monthly profits for Retailer Agents with different decision engines - best run in 5 attempts for medium demand elasticity.

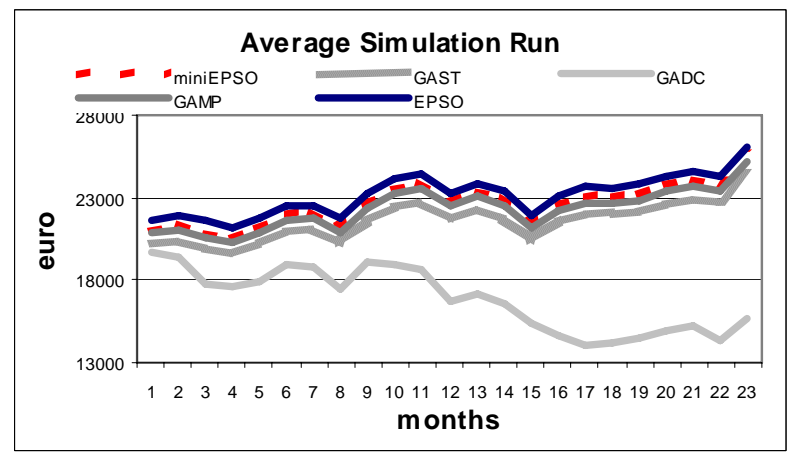

Fig. 7. Average of 5 runs for the evolution of monthly profits for Retailer Agents with different decision engines - medium demand elasticity. 


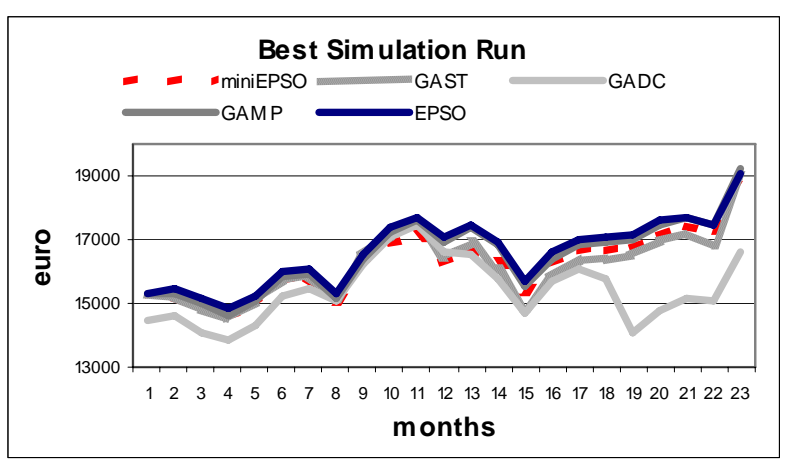

Fig. 8. Evolution of monthly profits for Retailer Agents with different decision engines - best run in 5 attempts for high demand elasticity.

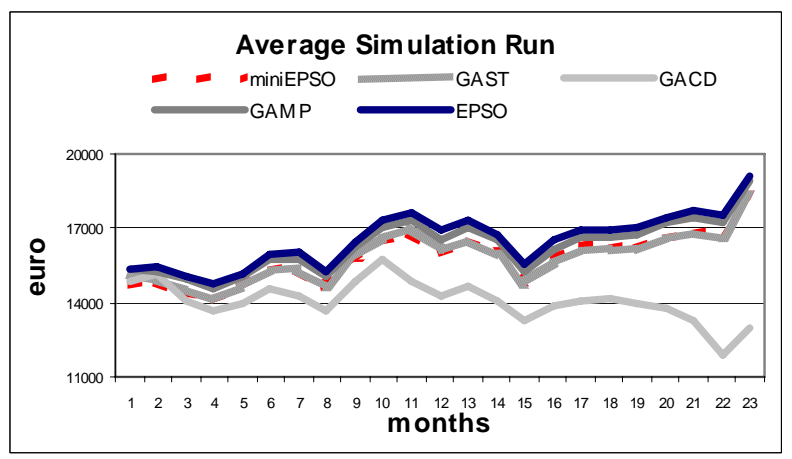

Fig. 9. Average of 5 runs for the evolution of monthly profits for Retailer Agents with different decision engines - high demand elasticity.

This is true in general even if we consider an EPSO variant with half the population (thus with less exploring power). As an accessory result, we also see that the deterministic crowding selection method is, in this complex problem, quite unsuccessful. We also see that EPSO provides not only the best single result in every simulation but also the best average. There is no interesting trade off here between expected value and variance of the results.

The advantage of EPSO is clearer in the scenarios with low elasticity in consumer response. This may be explained because this problem is probably more difficult, demanding continuous change in decision variable values such as prices. In the high elasticity scenarios, the solutions at every time step are closer to one another because the dynamics of the market generate a tighter control and the feedback reaction of consumers tends to keep the equilibrium. Therefore, all methods tend to discover similar decisions in this case.

In EPSO, contrary to GA, the recombination operation is not neutral: it also helps the convergence to the optimum, as proved by the classic PSO method. This may explain its superior performance.

\section{CONCLUSION}

The paper has presented comparison results from simulation experiments involving three scenarios and 5 distinct meta-heuristic models acting as decision engines inside Retailer Agents, from an intelligent agent platform for multi-energy market simulation

The fact that extremely complex behavior emerges from the interaction of agents, without any explicit definition of such behavior, speaks in favor of the agent technology and the model developed. This work explores a fruitful path for research not only in the application of the technology to energy systems but also in the behavior of energy markets.

Interesting conclusions may be derived from the comparison of the performance of distinct evolutionary meta-heuristics, optimizing the profits gained by a retailer during a given period, when facing competition. The evolution of profits depended on consistent "good guesses" or good decisions made regularly by the Retailer Agents. To achieve this, we have incorporated in Retailer Agents a decision process based in a meta-heuristic optimization, acting on a "second-order simulation" of the market inside the agent, before an actual move is done.

It became obvious from observations that an evolutionary method borrowing the movement rule from PSO and using it as an adaptive recombination operator leads to better results than any of the three experimented Genetic Algorithm variants. This method is called EPSO, for Evolutionary Particle Swarm Optimization, and is quite promising.

\section{REFERENCES}

[1] Gerald B. Sheblé, “Agent Based Economics”, Power Systems Restructuring: Engineering and Economics, Kluwer, 1998.

[2] FIPA - Foundation for Intelligent Physical Agents http://www.fipa.org/

[3] Fabio Bellifemine, Agostino Poggi and Giovanni Rimassa, "JADE A FIPA-compliant Agent Framework”, Proceedings of PAAM'99, London, April 1999, pp.97-108.

[4] JADE - Java Agent Development framework - http://jade.tilab.com/

[5] “ARCHON, an architecture for multi-agent systems”, Wittig T. Ed., Ellis Horwood, 1992, ISBN 0-13-044462-6

[6] L. Tesfatsion, “Agent-based Computational Economics: Growing Economies from the Bottom Up”, Artificial Life, 8:55--82, 2002.

[7] Waldemer Kamrat, "Modeling the Structure of Local Energy Markets”, IEEE Computer App. in Power, page 30-35, April 2001.

[8] V. Miranda and N. W. Oo, "Evolutionary Algorithms and Evolutionary Particle Swarms (EPSO) in Modeling Evolving Energy Retailers”, Proceedings of PSCC 2005 - Power Systems Computation Conference, Liège, Belgium, Aug 2005

[9] H. Mori and Y. Komatsu, "A Hybrid Method of Optimal Data Mining and Artificial Neural Network for Voltage Stability Assessment”, Proceedings of IEEE St. Petersburg PowerTech, Russia, June 2005

[10] N. W. Oo and V. Miranda, "Multi-energy Retail Market Simulation with Intelligent Agents", Proceedings of IEEE St. Petersburg PowerTech Conference, Russia, June 2005

[11] V. Miranda and N. Fonseca, "EPSO - Best-of-Two-Worlds MetaHeuristic Applied to Power System Problems”, Proceedings of IEEE WCCI/CEC - World Conference on Computational Intelligence/Conference on Evolutionary Computing, Honolulu, Hawaii USA, May 2002

[12] V. Miranda and N. Fonseca, "EPSO - Evolutionary Particle Swarm Optimization, a New Algorithm with Applications in Power Systems", Proceedings of the IEEE Transmission and Distribution Asia-Pacific Conference 2002, Yokohama, Japan, Oct 2002

\section{BIOGRAPHIES}

Naing Win Oo is a researcher at the Power Systems Unit of INESC Porto Institute of Engineering in Systems and Computers of Porto, Portugal. He obtained his M.Eng. degree in Power System Engineering from AIT - Asian Institute of Technology, Thailand, in 1998. Presently, he is in the PhD program in the University of Porto and developing his thesis work at INESC Porto, and his interests include energy markets, power system planning and control, evolutionary computation and artificial intelligence applications to power systems.

Vladimiro Miranda received his graduation, Ph.D. and Agregado degrees from the Faculty of Engineering of the University of Porto, Portugal (FEUP) in 1977, 1982 and 1991, all in Electrical Engineering. In 1981 he joined FEUP and currently holds the position of Professor Catedrático (Full Professor). He is also currently Director of INESC Porto - Institute of Engineering in Systems and Computers of Porto, Portugal. He has authored many papers and been responsible for many projects in areas related with the application of Computational Intelligence to Power Systems. 